Copyright (C) 2017 Johns Hopkins University Press. This article first appeared in PORTAL: LIBRARIES AND THE ACADEMY, Volume 17, Issue 3, July, 2017, pages 507-527.

\title{
No Uniform Culture: Patterns of Collaborative Research in the Humanities \\ Jennie M. Burroughs
}

\begin{abstract}
This study uses a campus scholarly networking and expertise system to examine trends in coauthorship in order to measure the prevalence of deep collaboration and readiness for team research. Bibliometric analysis of publishing patterns in four departments in the humanities shows significant differences in the rate of coauthorship by type of publication, by department, and by career phase. The data also show that coauthored research has become more common over time. Rather than a uniform culture, there is notable variation in collaborative practices between departments. This finding suggests that a more granular approach may be needed to incorporate humanities perspectives in team research.
\end{abstract}

\section{Introduction}

Academia is experiencing a growing emphasis on team research and interdisciplinary collaborative research. This increase can be seen in publishing trends, in the grant requirements from federal funding agencies, and in the "grand challenges" initiatives setting ambitious but attainable goals at many universities. Many experts see interdisciplinary research as gathering the requisite expertise to tackle major societal issues and to advance discovery in creative directions.

Given the challenges inherent in this form of research, academic institutions are developing financial and policy incentives and infrastructure to support the advancement of this 
work. While numerous studies over the past 20 years demonstrate that science, technology, engineering, and mathematics (STEM) and medical research practices have become progressively more collaborative, in fact participating in a "collaboration imperative," there have been fewer studies on collaborative practices in the humanities. ${ }^{1}$ Where do humanists fit in the growing trend toward interdisciplinary research? Culture, history, and modes of communication all influence the complex issues addressed in "grand challenges" initiatives and other team research efforts. As a result, it is essential that humanities perspectives be brought to bear in addressing these critical questions. It is more important than ever to dismantle the "two cultures" dynamic that divides the sciences from the humanities. ${ }^{2}$

How inclined are humanities scholars to collaborate on research or to participate in team research initiatives, and what can libraries do to foster the inclusion of humanists in team research? Do traditional assumptions about the lack of formal collaboration in the humanities remain true, or is humanities collaboration on the rise? There are some assumptions that growing experimentation with digital humanities methods aligns with increased openness to collaboration and interdisciplinary research. As libraries reconceive their support for the full life cycle of knowledge creation and expand their partnership roles in academic environments to encourage research collaboration, it is valuable for libraries to identify humanists or humanities cohorts ready to participate in team research. As a first step, this author conducted a pilot study using bibliometric indicators of collaboration at the University of Minnesota Twin Cities. The study used the campus scholarly networking and expertise system, supported by the University Libraries, to identify prevalence of coauthorship and coauthorship patterns with the hope of supporting a developing interest in digital humanities and team research on campus. The results uncovered some significant correlations and complexities in humanities collaboration, suggesting 
a more granular approach to outreach, areas needing further research, and issues worthy of consideration for libraries that seek a role in shaping interdisciplinary research in university settings.

\section{Background}

Numerous studies have documented the growth of cooperative research and team science. Stefan Wuchty, Benjamin Jones, and Brian Uzzi examined 19.9 million research articles in Web of Science, an online citation indexing service, including articles in the humanities for 1975 to 2000. They looked for articles with more than one author and confirmed a rise in collaborative research in all fields of study. ${ }^{3}$ The Research Information Network, a policy group in the United Kingdom, examined humanities information practices through interviews and focus groups. It described a rise in formal and systematic collaboration in humanities research over the past 10 to 15 years, which it credits to new funding and technological opportunities. ${ }^{4}$ Lynne Siemens, Richard Cunningham, Wendy Duff, and Claire Warwick propose that this shift reflects a "realization that the research questions addressed by academics are becoming more complex and technologically sophisticated" and requires a team-based approach to draw on greater capacity and breadth of skills and knowledge. ${ }^{5}$ A member of the Research Information Network study observed that cross-disciplinary collaborations, in particular, allow "participants to derive much more complex and novel outputs when they tackle research questions from a variety of methodological as well as theoretical standpoints." ${ }^{\circ 6}$ Beyond examining trends in incidence, the Wuchty analysis found that work produced by teams was more highly cited than work by individual authors in all broad research areas, including the humanities. ${ }^{7}$ This finding indicates that collaborative research may garner deeper regard and influence than solo-authored work. 
Universities place increased emphasis on collaborative research for just such reasons. Looking at a specific example on a local level, the University of Minnesota's strategic plan, approved in 2014, emphasizes research and curricular efforts that address "grand challenges of compelling public interest," which necessarily require "expertise from multiple fields of knowledge" for success. ${ }^{8}$ The plan cites identifying and addressing barriers and risks associated with interdisciplinary research as critical to this goal. The university lists promotion and tenure standards as an existing barrier to interdisciplinary work, and the strategic plan expressly includes the suggestion of reconsidering the treatment of multiple-author publications as one way to lower this barrier. ${ }^{9}$ Several studies of collaborative work have also noted these research barriers and risks. In a study by Jenny Lewis, Sandy Ross, and Thomas Holden, 70 percent of humanities scholars cited "discipline tradition" as a factor impacting their decision to publish individually or collaboratively, with the implication that collaboration is less common in arts and humanities departments. ${ }^{10}$ Scholars early in their careers have expressed concern about the impact of interdisciplinary research on their academic prospects on the tenure track. ${ }^{11}$

So, if collaboration is increasing in the humanities but also faces ongoing challenges, how can we gauge the readiness of humanities scholars to take part in team research? Surveys and interviews of humanities scholars convey a perception of collaboration and interdisciplinarity as core values. In an Andrew W. Mellon Foundation-funded study at the University of Minnesota in 2006, 68.5 percent of College of Liberal Arts (CLA) faculty reported that they work collaboratively. The humanities researchers who indicated that they work collaboratively described joining forces both with colleagues at other institutions (52.3 percent) and with colleagues outside their discipline (40.9 percent). ${ }^{12}$ Lisa Mooney Smith writes that "the majority of arts and humanities academics actually perceive their predominant style of 
collaboration as interdisciplinary, and increasingly transdisciplinary." She notes the potential benefit to team dynamics from including these inherently multidisciplinary scholars in collaborative, radically interdisciplinary teams. ${ }^{13}$

The literature on team research suggests some indicators of collaboration readiness that can help with identifying humanist partners for interdisciplinary research. Daniel Stokols, Shalini Misra, Richard P. Moser, Kara L. Hall, and Brandie K. Taylor note the critical importance of looking at past successful collaboration as an indication of team members' readiness for future interdisciplinary teams. ${ }^{14}$ Jonathon Cummings and Sara Kiesler found similar results regarding the positive impact of prior collaborative experience. ${ }^{15}$ These findings provide a major rationale for examining humanists' past collaboration practices to gauge readiness for participating in future, high-impact team research.

What is an appropriate metric to gauge past collaborative practice? Willard McCarty notes the spectrum of collaboration in the humanities ranges "from broad, indirect and tacit indebtedness to explicitly joint work." ${ }^{, 16}$ Looking to coauthorship as an indicator may be problematic because scholars in the humanities "have tended to be physically alone when at work because their primary epistemic activity is the writing, which by nature tends to be a solitary activity." ${ }^{17}$ However, due to the primacy of writing, coauthorship can be read as evidence of a substantive collaboration for humanists. John Unsworth sees collaboration rising to a new level of significance in the humanities due to the complexity and size of digital humanities (DH) work, which includes scholarship enabled by digital methods as well as scholarship about digital technology and culture. But Unsworth acknowledges that the increase in collaboration is "mostly happening beneath the radar on which authorship blips." "18 That said, he perceives the humanities as shifting from a cooperative model, where individuals draw on the work of other 
individuals, to a collaborative model, where work and scholarship is produced jointly and cannot be attributed to a single author. ${ }^{19}$ Sylvan Katz and Ben Martin wrote a seminal study addressing the issue of metrics for research collaboration, observing that "for decades the multiple-author publication, frequently referred to as a co-authored publication, has been used as a basic counting unit to measure collaborative activity." 20 Acknowledging that coauthorship is an imperfect indicator, there is value to using this data point because it is "invariant and verifiable" and serves as a practical means for analyzing larger samples that can provide more statistically significant results. ${ }^{21}$ Using bibliometric indicators may not capture all instances of humanities collaboration, but teamwork that rises to the level of coauthorship is a verifiable indicator of substantive collaboration and joint effort.

Recent studies using bibliometric data describe varying levels of humanities collaboration. Wuchty and his coauthors note that single authors produce 90 percent of articles in the arts and humanities. ${ }^{22}$ A study at the University of Melbourne in Australia covering 2001 to 2005 found that 13 percent of books, 21 percent of book chapters, and 19 percent of articles in the arts had two or more authors. ${ }^{23}$ Lisa Spiro examined existing assumptions about the prevalence of collaborative effort in digital humanities work by reviewing coauthorship patterns in two leading journals, American Literary History (ALH), covering American literature and culture, and Literary and Linguistic Computing (LLC), representing DH, for the period 2004 to 2008. She reported, "Whereas 5 of 259 (1.93\%) articles published in ALH—about one a yearfeature two authors (none had more than two), 70 out of $145(48.28 \%)$ of the articles published in LLC were written by two or more authors." 24

Julianne Nyhan and Oliver Duke-Williams are more skeptical about collaboration and DH. Their examination of publication patterns over a longer period in LLC (1986 to 2011) and in 
Computers and the Humanities (1966 to 2004) noted that single-authored papers predominate, with 69 percent of papers in Computers and the Humanities and 65 percent of papers in LLC produced by single authors. ${ }^{25}$ The analysis reveals a further nuance by observing that "there is a relatively small cadre of authors who co-publish with a wide set of other authors, and a longer tail of authors for whom co-publishing is less common." ${ }^{26}$ The study by Nyhan and DukeWilliams affirms the notion that DH work features more coauthorship than other forms of humanities scholarship but suggests that a smaller pool of scholars drive this difference. It also intimates the value of looking at those prime collaborators as leaders in team research. A study at Cornell University in Ithaca, New York, analyzed coauthorship networks using the university's VIVO scholarly networking and expertise system, which relies heavily on publication information, as the data source to identify likely collaborative "brokers" and the most connected researchers on campus. ${ }^{27}$ That study provided a key research precedent that informs the methodology employed in this Minnesota-based project.

One way the University of Minnesota Libraries supports collaborative and interdisciplinary research is through stewardship of the Experts@ Minnesota tool. The libraries began partnering with the Office of the Vice President for Research in 2012 to cosponsor an implementation of SciVal, a scholarly interaction tool marketed by Elsevier, branded locally as Experts@Minnesota. Experts@ Minnesota is a research networking system that displays faculty members' areas of expertise based on their grants and publications, articulates campus coauthor networks, identifies potential partners based on each scholar's "research fingerprint" (that is, areas of research interest based on publication history and Elsevier-generated thesauri), and finds relevant sources of funding based on areas of interest. The intention of the Experts@Minnesota tool is to highlight expertise at the university and to connect scholars across departmental and 
disciplinary boundaries. Libraries serve as key connectors on campus and are frequent partners in digital humanities work, which is often interdisciplinary in nature. Libraries might utilize the data in these types of systems to identify potential collaborative partners and examine coauthorship patterns for departments and for scholars in different career phases to identify areas where administrative or departmental norms may inhibit collaborative work and warrant further outreach and support.

The growing complexity of academic research and institutional drives to explore "grand challenges" suggest that deep collaboration will become increasingly common for humanities scholars. To that end, while they are an imperfect measure, coauthorships in the humanities are a reasonable stand-in for closely cooperative work styles, given the epistemic nature of writing in the humanities. Based on previous studies of team research, examining patterns of coauthorship may point to individuals with significant earlier collaborative expertise and indicate potential partners ready for team research efforts. These team-ready individuals may serve as valuable influencers in their academic departments and affect disciplinary norms.

\section{Methodology}

To examine collaborative readiness, the author conducted a bibliometric analysis examining quantifiable indicators hosted in the Experts@Minnesota system. At the time of the project, the publication data source was the Scopus database, a large, multidisciplinary citation and abstract index. Scopus is particularly strong in science coverage and focuses primarily on journal publications. It contains considerably less data on social science and humanities publications, and the majority of journal coverage for the humanities goes back to only 2002. The University of Minnesota Libraries augmented the profiles of CLA faculty by adding entries based on faculty curricula vitae (CVs), amounting to nearly 30,000 citations for books, book chapters, articles not 
indexed by Scopus, book reviews, creative works (for example, poems or short stories), and performances. The data added by the University of Minnesota Libraries to the Experts@Minnesota system to more fully reflect the scholarly output of researchers in the arts and humanities enable analysis of collaboration that goes beyond journal articles.

However, there are challenges to using the hand-entered data stemming from faculty CVs. On a few occasions, for example, authors indicated that a work was coauthored or coedited without listing the partners. In such cases, library staff entering data note the presence of coauthors because there were no specific authors to add.

The Experts@ Minnesota tool lists 32 departments within CLA, comprising 542 faculty members. To demonstrate proof of concept, a pilot study of four departments was used to develop a common data structure, uncover data compilation issues, and test analytic processes. The study focused on the Departments of English; Gender, Women, and Sexuality Studies (GWSS); History; and Writing Studies. These departments were chosen because faculty members in these departments have been among the most active in local DH programming, teaching, and research. The assumption was that these departments might provide more examples of collaboration to review and study.

At the time of this project's inception, there were 96 faculty members total in the four pilot departments, and 89 faculty members are represented in the pilot data: 30 from English, 8 from GWSS, 40 from History, and 11 from Writing Studies. While all faculty members at the University of Minnesota-Twin Cities are included in Experts@ Minnesota by default, some faculty members have opted to remove their information from the system. In the pilot set of departments, five faculty members in the pilot set (three in English, one in History, and one in Writing Studies) had opted out of the Experts system. In addition, two faculty members in the 
pilot departments (one in History and one in English) had no publications listed in the Experts@Minnesota system when the project began and were excluded from the pilot data.

The data for the study were extracted via the Experts@Minnesota application program interface (API), which returns Extensible Markup Language (XML) based on the queries. The author extracted the XML by publication type-Book, Book chapter, Article (indicating an article hand-entered from faculty CVs), and Scopus publication-and by Expert ID, an identification number assigned by the system. Different fields were used for each publication type, which made a bulk export solely by individual Expert ID difficult and required conversion into a shared data frame (that is, a structure common to all publication types). The data frame included the following fields:

\section{Expert ID}

Name (University of Minnesota author for the record)

Department name

Department ID

Organization

Rank

Publication ID (assigned by the system)

Title 1 (primary title: article title; chapter title; or book title)

Title 2 (source title: journal title; the book containing the chapter; or blank for book entries)

Date (Year)

Volume/Issue (data combined from separate fields and retained for disambiguation)

Pages (retained for disambiguation)

Authors (containing all authors of the publication, including the University of Minnesota author and any coauthors) 
The study author added data reflecting the publication type, number of authors, and whether an entry had more than one author. Publications written by multiple authors included in the pilot set would receive an entry for each author-for example, if two Writing Studies authors included in the pilot set wrote an article together, there would be two data entries for that article, one entry with the first author's Expert ID and accompanying information, and the other entry with the second author's Expert ID and accompanying information.

In cleaning the data, the study author made additional choices about how to treat different data elements. For the purpose of the project, when faculty members wrote or edited a book, fellow editors were classed as coauthors; for book chapters, additional authors on the chapter were treated as coauthors, and editors of the book were ignored. Book reviews were classed as a separate publication type, "Reviews," due to the distinctiveness of the publication type and the ease of recognizing these works through indications in the title field. However, creative writing works were not handled as a separate type due to the difficulty of distinguishing these works from others classed as articles in Experts@Minnesota. Instead, the analysis examined differences between creative writing and noncreative writing faculty as a means of differentiating these types of works. The author reviewed the data for duplications, removing entries hand entered in 2013 when those publications had subsequently been indexed and imported into Experts@ Minnesota from Scopus. The Scopus version of the record was retained due to the completeness of information. This compilation and cleaning resulted in a file of 3,597 publication entries: 1,509 articles (which includes short stories and poems), 349 books, 872 book chapters, and 867 reviews. 


\section{Humanist Trends at the University of Minnesota}

The publications for the four departments cover a span of 55 years, a range of publication types, and members with differing academic status. Given the data available in the Experts@ Minnesota system, it is possible to look at whether publication type, department, or rank correlates with collaboration habits.

Most bibliometric studies of the humanities to date have relied solely on journal publications, and the Experts data permit exploration of whether the type of publication makes a difference in coauthorship status. With the expansion of the data to include books and chapters, in addition to journal articles, it is possible to assess whether the inclusion changes the overall picture of humanities collaboration.

Examining the influence of departmental home, the University of Minnesota Mellon study reported that the difficulty of evaluating collaborative work and a lack of disciplinary or administrative rewards and incentives were significant barriers to both collaborative and interdisciplinary work. ${ }^{28}$ Academics frequently cite promotion and tenure issues as barriers to collaborative research in the humanities, so it is worthwhile to examine if these barriers are reflected in the coauthorship patterns of academic departments. In short, does one's home department make a difference in the amount of coauthoring one does? While it is tempting to consider department and discipline equivalent, the faculty members interviewed in the Mellon study pointed out that "their departmental colleagues engage in very different kinds of work and are sometimes joined by somewhat tenuous or structural ties. ${ }^{29}$ Those structural ties have significant bearing on promotion and tenure questions, however, so it seems particularly important to examine department-level trends. 
There are competing hypotheses with regard to rank. One hypothesis is that faculty members with established careers will have had more time and opportunity to build connections, resulting in a greater number of coauthorships. A competing argument is that, as collaboration becomes more prevalent in the humanities, new faculty members will be more inclined to collaborate as a reflection of changing disciplinary norms and will demonstrate a higher rate of coauthorship. A factor complicating this analysis is how much the overall shift toward increased coauthorship in the humanities affects the interpretation of the influence of rank.

The coauthorship rate for the set of four departments, 11.9 percent, mirrors the low end of recent bibliometric analyses of humanities coauthorship. However, the numbers shift as one examines publication types, and the differences between departments tell a story of greater nuance.

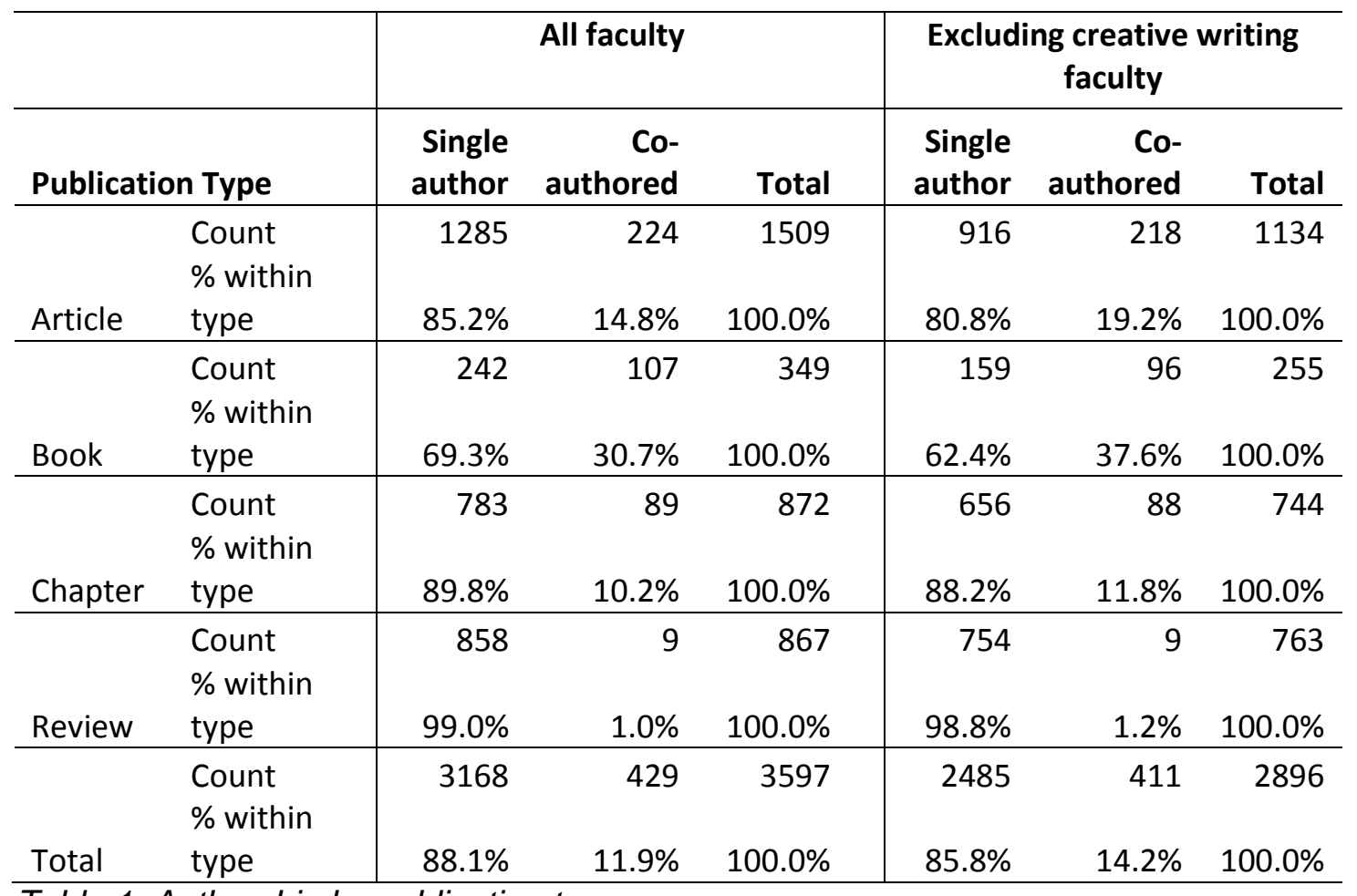

Table 1. Authorship by publication type 
There are significant differences in the rate of coauthorship for different publication types. Scholarly reviews are almost entirely solo-authored, reflecting the work of close examination of a fellow scholar's work, often a book. By contrast, books are coauthored or coedited at a much higher rate (30.7 percent). If one removes scholarly reviews from the analysis, the overall coauthorship rate for the set increases to 15.4 percent.

The data also reveal that the rate of coauthorship varies by department to a significant degree, indicating that a monolithic view of "the humanities" does not accurately reflect the differences in departmental norms.

\begin{tabular}{|c|c|c|c|c|}
\hline Departmen & & $\begin{array}{l}\text { Single } \\
\text { author }\end{array}$ & $\begin{array}{r}\text { Co- } \\
\text { authored }\end{array}$ & Total \\
\hline & Count & 1230 & 67 & 1297 \\
\hline \multirow[t]{2}{*}{ English } & $\%$ within dept. & $94.8 \%$ & $5.2 \%$ & $100.0 \%$ \\
\hline & Count & 198 & 49 & 247 \\
\hline \multirow[t]{2}{*}{ GWSS } & $\%$ within dept. & $80.2 \%$ & $19.8 \%$ & $100.0 \%$ \\
\hline & Count & 736 & 125 & 861 \\
\hline History & $\%$ within dept. & $85.5 \%$ & $14.5 \%$ & $100.0 \%$ \\
\hline Writing & Count & 146 & 179 & 325 \\
\hline \multirow[t]{2}{*}{ Studies } & $\%$ within dept. & $44.9 \%$ & $55.1 \%$ & $100.0 \%$ \\
\hline & Count & 2310 & 420 & 2730 \\
\hline \multirow[t]{2}{*}{ Total } & $\%$ within dept. & $84.6 \%$ & $15.4 \%$ & $100.0 \%$ \\
\hline & Value & $d f$ & Sig. & \\
\hline $\begin{array}{l}\text { Chi-square } \\
\text { Table 2. Co }\end{array}$ & $\begin{array}{r}501.634 \\
\text { Ithorship by dep }\end{array}$ & ent ex & $\begin{array}{c}0.000 \\
\text { uding revi }\end{array}$ & \\
\hline
\end{tabular}

Noting that scholarly reviews are primarily solo-authored, it is useful to examine department coauthorship trends both including and excluding these reviews. If one excludes reviews, the English Department ranks well below the average coauthorship rate of the overall set, with only 5.2 percent of publications having a coauthor. On the other end of the spectrum, 
Writing Studies publications are coauthored 55.1 percent of the time, well above the average coauthorship rate of other bibliometric studies and closer to the self-reported rate of collaboration. Removing scholarly reviews from the departmental percentages makes a notable difference to the coauthorship rate for History and a small difference for English. In the pilot set of publications, 38.5 percent of History publications and 18.7 percent of English publications are reviews, compared with GWSS (6.8 percent) and Writing Studies (3.3 percent). Including reviews in the coauthorship rate by department would reduce the coauthorship rate of History to 8.9 percent and that of English to 4.4 percent.

There are additional factors to consider when comparing the coauthorship rates of departments. First, it is important to look more deeply at the low numbers of coauthorship for English and recognize that they could result from including creative writing faculty, who are more likely solo authors. While it is challenging to parse the article field for poetry, short stories, and other creative works, it is more feasible to identify creative writing faculty based on departmental websites. Distinguishing the work of creative writing faculty reveals more about the range of academic work in humanities departments. The data set includes seven creative writing faculty, who are responsible for 701 of the publications in the overall data set (19.5 percent of the total publications). These faculty members coauthor at a significantly lower rate than their English Department peers: 2.6 percent overall. Even excluding scholarly reviews, 3 percent of creative writing faculty publications are coauthored. Removing their publications from the overall set (and excluding reviews) raises the coauthorship rate for English Department publications to 7 percent (compared to the earlier noted 5.2 percent) and increases the overall coauthorship rate for the data set to 18.8 percent (compared to 15.4 percent). There is also an impact on the coauthorship rate by publication type, as indicated in Table 1. Removing works by 
creative writing faculty from the overall set raises the coauthorship rate noticeably for articles (19.2 percent, compared to 14.8 percent) and books (37.6 percent, compared to 30.7 percent) and by a smaller amount for chapters and reviews.

The next critical question is whether particularly prolific faculty members skew the results toward greater or lesser amounts of coauthorship.

\begin{tabular}{|c|c|c|c|c|c|}
\hline Department & & $\begin{array}{r}\text { Co-authored } \\
\text { mean }\end{array}$ & $\begin{array}{r}\text { Percent Co- } \\
\text { authored }\end{array}$ & $\begin{array}{r}\text { Co-authored } \\
\text { mean w/o } \\
\text { reviews }\end{array}$ & $\begin{array}{r}\text { Percent Co- } \\
\text { authored }\end{array}$ \\
\hline & Mean & 0.058 & $5.8 \%$ & 0.075 & $7.5 \% *$ \\
\hline & $\mathrm{N}$ & 30 & & 30 & \\
\hline & Std. Deviation & 0.073 & & 0.085 & \\
\hline \multirow[t]{4}{*}{ English } & Std. Error of Mean & 0.013 & & 0.016 & \\
\hline & Mean & 0.146 & $14.6 \%$ & 0.149 & $14.9 \%$ \\
\hline & $\mathrm{N}$ & 8 & & 8 & \\
\hline & Std. Deviation & 0.188 & & 0.187 & \\
\hline \multirow[t]{4}{*}{ GWSS } & Std. Error of Mean & 0.066 & & 0.066 & \\
\hline & Mean & 0.095 & $9.5 \%$ & 0.129 & $12.9 \%$ \\
\hline & $\mathrm{N}$ & 40 & & 40 & \\
\hline & Std. Deviation & 0.134 & & 0.154 & \\
\hline \multirow[t]{3}{*}{ History } & Std. Error of Mean & 0.021 & & 0.024 & \\
\hline & Mean & 0.547 & $54.7 \%$ & 0.567 & $56.7 \%$ \\
\hline & $\mathrm{N}$ & 11 & & 11 & \\
\hline Writing & Std. Deviation & 0.181 & & 0.178 & \\
\hline \multirow[t]{4}{*}{ Studies } & Std. Error of Mean & 0.055 & & 0.054 & \\
\hline & Mean & 0.143 & $14.3 \%$ & 0.166 & $16.6 \% *$ \\
\hline & $\mathrm{N}$ & 89 & & 89 & \\
\hline & Std. Deviation & 0.201 & & 0.207 & \\
\hline Total & Std. Error of Mean & 0.021 & & 0.022 & \\
\hline
\end{tabular}

${ }^{*}$ Excluding creative writing faculty raises English Department co-authorship mean to $8.4 \%$ and the overall co-authorship mean to $17.7 \%$.

Table 3. Comparison of author means by department

The study author coded each publication in the pilot set as single-authored (0) or coauthored (1), then calculated coauthorship means for each author in the set, both including and excluding scholarly reviews. This averaging normalized the contribution of faculty members to 
the set and accounted for the possibility that a particularly prolific faculty member might skew the department average overall. These means were then compared at a departmental level. The differences between the departments remained, but there were some changes to the coauthorship rates noted earlier. Overall, the analysis comparing author means shows that prolific faculty inclined toward solo authorship skewed the results of the "publication mean" toward lower levels of coauthorship. This skewing is indicated by a total author mean of 0.166 , or 16.6 percent, compared with the mean of the publication set at 15.4 percent. This difference in modes of calculating coauthorship means is mirrored in the data for English and Writing Studies (to a small extent). Excluding creative writing faculty from the set raises the overall coauthorship mean to 17.7 percent and to 8.4 percent for the English Department. The inverse effect is found with History and GWSS, where prolific faculty in those departments inclined toward coauthorship skew analysis to give an impression of higher levels of coauthorship across the department. Using the author mean, History Department faculty worked with coauthors 12.9 percent of the time; the publication mean indicates that 14.5 percent of departmental publications were coauthored (again, excluding reviews).

Looking at the effect of time and rank (or career phase) on the rate of coauthorship is complicated. The publications in the data set range from 1959 to 2014 . The cumulative average of coauthorship does not take into account whether there are differences between coauthorship rates in the early part of the time range and those in the later part of the time range (for example, whether the overall rate of coauthorship differs significantly in each decade). It is also worth questioning whether a mean rate of coauthorship accurately captures faculty tendencies or whether it elides differences between early-career and later-career behavior. 
To address the first question, whether there are overall differences in the rate of coauthorship in the humanities over time, the study author clustered articles by the decade in which they were published and then compared the mean rates of coauthorship for each decade.

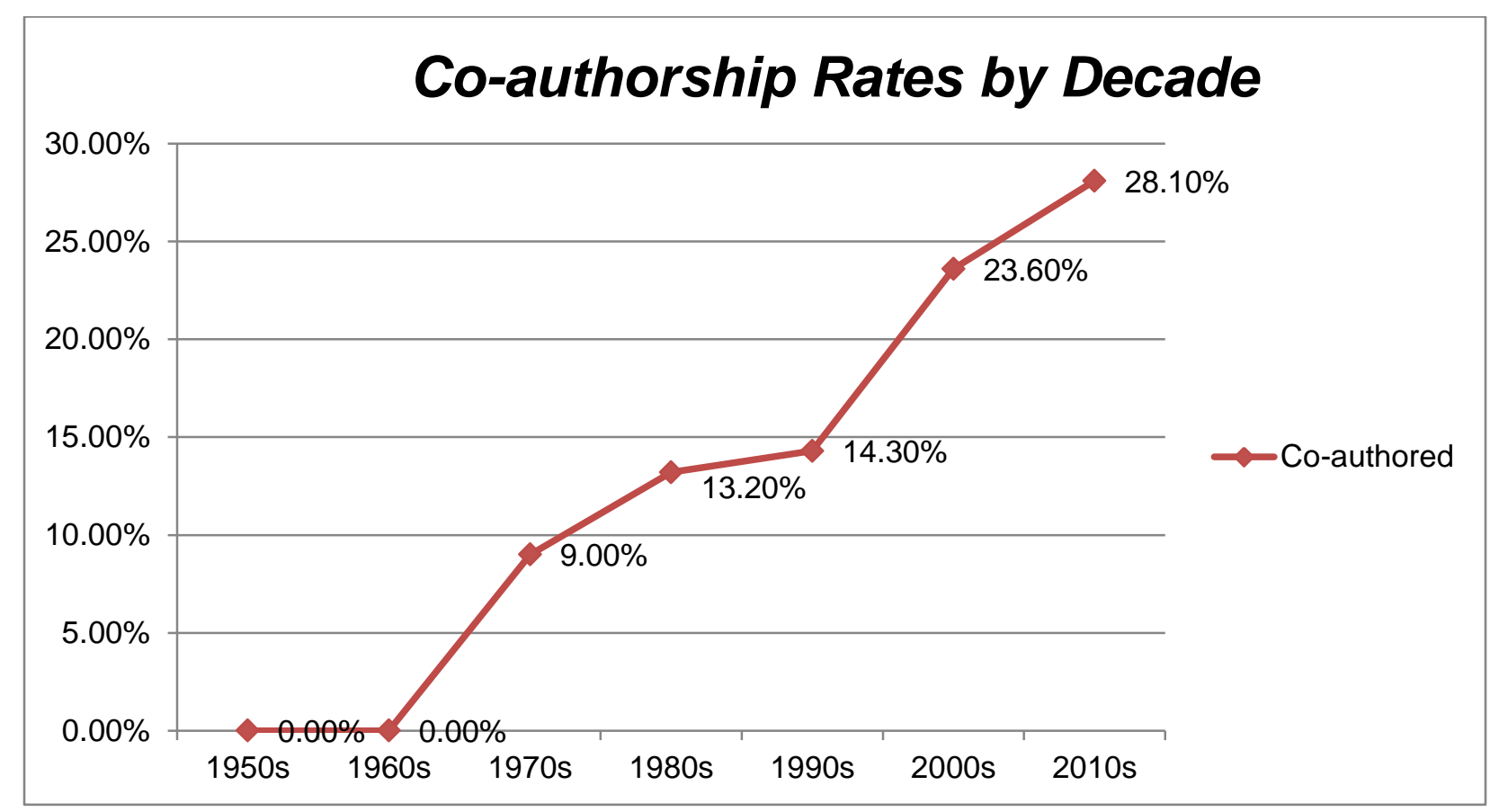

Figure 1. Co-authorship rates by decade, excluding scholarly reviews, excluding creative writing faculty publications

The data show a significant increase in the rate of coauthorship over time. The coauthorship rate over the past 15 years (that is, 2000 to 2014) is higher than the overall average, indicating a steady increase in publication collaboration. This suggests that humanists indeed collaborate on research more frequently than they have in the past. However, it is also worth noting that this is a smaller rate than that reported by Spiro or by Nyhan and Duke-Williams in their reviews of DH journals. ${ }^{30}$

To address the issue of rank and career phase, the study author generated the coauthorship means for the first five works and the last five works of each author in the pilot set to serve as a proxy for typical behavior in earlier and later career stages. This is an imperfect 
measure due to the range of time covered by each set of five publications, but the first five publications for each individual cover a mean of 5.79 years and the last five publications cover a mean of 3.52 years.

Std.

\begin{tabular}{lrrrr} 
& Mean & N & Deviation & Std. Error Mean \\
\hline First 5 Publications Mean & 0.124 & 71 & 0.228 & 0.027 \\
& & & & \\
Last 5 Publications Mean & 0.215 & 71 & 0.309 & 0.037 \\
\hline
\end{tabular}

Paired Differences

The author used a statistical technique called a paired samples $t$-test, comparing matched pairs of means for the first five and last five publications for each author, to determine whether the changes in coauthorship rates were statistically significant rather than a difference that occurred because of random chance. The $t$-test suggests a significant difference in the rate of coauthorship over the course of an academic career.

The data in Table 4 show a coauthorship rate of 12.4 percent for early publications, compared with 21.5 percent for later publications. It should be noted that this comparison included scholarly reviews, which have an overall lower rate of coauthorship. The $t$-test does not include the newer faculty who had five or fewer publications in the set (and thus did not have a "last five" publications for comparison). The mean rate of coauthorship in the first five publications for this subset of new faculty is 4.2 percent, suggesting that the assumption that 
newer faculty are more accustomed to collaboration and coauthoring is incorrect. Table 4 excludes creative writing faculty from the analysis. A paired samples $t$-test comparing the coauthorship of the first five publications with the last five publications produced by creative writing faculty failed to yield a statistically significant difference within the set, suggesting that coauthorship tendencies are more consistent over time for creative writing faculty.

\begin{tabular}{llrr}
\hline & & $\begin{array}{r}\text { First 5 } \\
\text { Publications } \\
\text { Mean }\end{array}$ & $\begin{array}{r}\text { Last 5 } \\
\text { Publications } \\
\text { Mean }\end{array}$ \\
\hline \multirow{2}{*}{ English } & Mean & 0.076 & 0.076 \\
& N & 21 & 21 \\
GWSS & Mean & 0.148 & 0.223 \\
\hline \multirow{3}{*}{ History } & N & 0.029 & 0.257 \\
& Std. Deviation & 7 & 7 \\
& Mean & 0.076 & 0.299 \\
\hline Writing Studies & N & 0.063 & 0.127 \\
& Std. Deviation & 32 & 32 \\
& Mean & 0.118 & 0.202 \\
\hline Total & N & 0.455 & 0.709 \\
& Mean & 11 & 11 \\
& N & 0.359 & 0.226 \\
\hline
\end{tabular}

Table 5. Comparison of co-authorship means for first 5 publications with last 5 publications by department, excluding creative writing faculty and authors without "last 5 " publication mean.

Comparing the first five and the last five publications at the department level shows some additional, interesting differences. As shown in Table 5, the pattern of the creative writing faculty is mirrored by the rest of the English Department faculty; the coauthorship tendencies are stable over time. By contrast, there is a significant jump between early- and late-career coauthorship in the History, GWSS, and Writing Studies departments. Each of these departmental comparisons demonstrates the great variation in collaboration practices in the large 
category of "the humanities." The differences highlight diversity in department norms. Given that the last five publications used as a proxy for later career trends would all have come from the last several years, it is hard to discern what has a greater effect: overall trends in humanities coauthorship or a greater ability (due to more connections or tenure-related security) to collaborate the further along a scholar is in his or her career.

\section{Implications for Libraries and Future Research}

There are some overall trends that can be discerned in this set of data. Publication type, departmental home, and timing of publication production all have a correlation with the rate of coauthorship. It also appears that there is an upward trend toward coauthorship over time. The departmental comparisons suggest that lumping these departments together under a broad disciplinary umbrella, "the humanities," misses the nuances in academic practices and serves to overlook pockets of greater collaborative tendencies within the humanities. For libraries and librarians seeking a role in advancing campus team and interdisciplinary research initiatives, it is worthwhile to conduct local investigations to determine humanities research practices at a more granular level. Assessing research outputs at the departmental level will serve to identify departments with norms that are favorable to collaboration, indicating readiness for team research initiatives. For libraries involved in building digital humanities programs, for instance, understanding which departments on a campus reward interdisciplinary research and multiauthor scholarly products can have an impact on outreach efforts and partnership development. Such investigation would also pinpoint departments that may require attention to change their culture, particularly with regard to credit for collaborative and interdisciplinary work in the promotion and tenure process, to create conditions conducive to team research. For libraries, this might mean highlighting standards in development for evaluation of digital and cooperative research, 
support for early-career scholars in demonstrating research impact, or systems for identification of potential research partners.

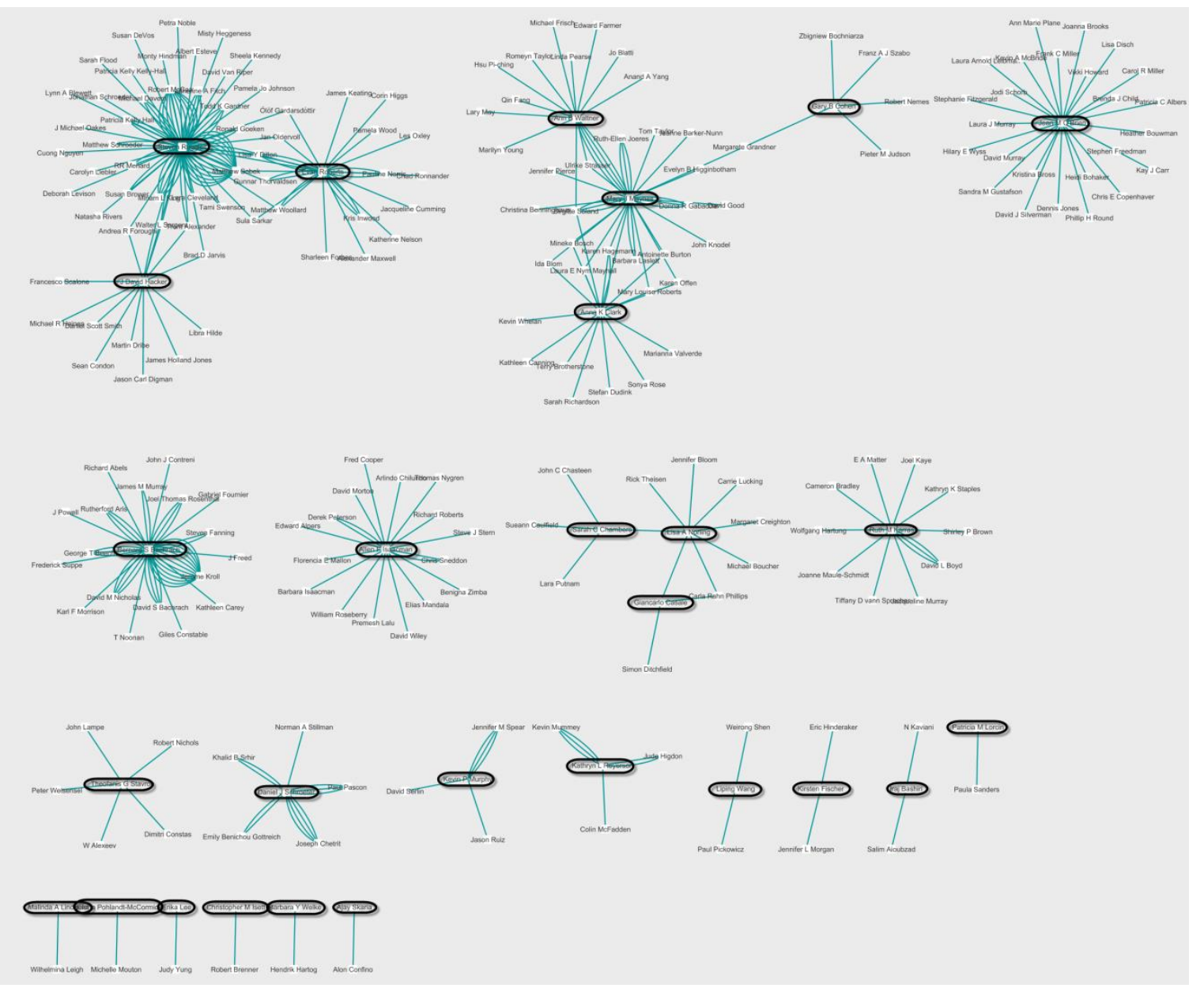

Figure 2. History Department co-author network map

In addition to statistical analysis of research outputs to identify patterns, it is useful to visualize authorship networks to emphasize the differences in collaboration practices in each department. This process has the added benefit for librarians of distinguishing specific research hubs (sites of frequent coauthorship) and which faculty members coauthor on a regular basis. This knowledge can inform library efforts to develop networking events and make matches between potential research partners. As an example of how this might look, author dyads were extracted from the data set to develop a network map of the History Department (Figure 2). The 
map shows the coauthorship relationships of department faculty (outlined with curved rectangles) and helps to highlight the work of a known research unit (the Minnesota Population Center, an interdisciplinary center for demographic research) and dyads that collaborate frequently. Such maps showcase high-frequency collaborators who are possible "collaboration brokers" and influencers. These individuals may help initiate collaborations and influence disciplinary norms toward greater receptivity to coauthorship. It would also be worthwhile to study the collaboration motivators of these individuals and their methods for developing research partnerships. Libraries visualizing the research networks on their campuses would gain the necessary specific information about humanities scholars who can bring successful collaboration practices to bear in a research team.

The pilot study of four CLA departments revealed some intriguing differences in departmental rates of coauthorship. With the consistent data frame in place and an established process for extracting and cleaning authorship data, it is feasible to analyze the remaining departments in CLA. This analysis would facilitate the identification of other high-collaboration departments and individuals. It would also be beneficial to do a similar analysis at other institutions to examine whether local department trends hold true elsewhere, indicating broader, disciplinary norms.

There are limits, however, to the information about collaborative research practices in humanities departments that can be gleaned from bibliometric and other quantitative analyses. The pilot data suggest that coauthorship is still not the dominant practice in humanities departments and that no individual scholar coauthors in all cases. In fact, only 7 out of 89 faculty members in the data set coauthor works more than 50 percent of the time. So, scholars regularly face decision points about whether to collaborate deeply on a research project or to pursue a 
project as a solo author. The limited information about collaborative drivers and research partner selection factors in humanities disciplines indicates it would be worthwhile to conduct a qualitative study of these issues. Interviewing scholars who collaborate frequently, as identified in data and network analysis, would provide more information about collaboration choices.

\section{Conclusion}

This study was conducted in response to growing organizational and scholarly incentives toward team research. A review of the literature signals a trend toward increasingly complex and interdisciplinary work that benefits from diverse perspectives to address the significant societal issues of the day. Reviewing the coauthorship trends of humanities faculty provides some indication of scholarly readiness to participate in this team research movement. The pilot study uncovered significant differences in coauthorship behavior in the four CLA departments. While coauthorship rates in English align with previous bibliometric analyses of humanities scholarship, the faculty members in Writing Studies coauthor works more than 50 percent of the time. This indicates that blanket assumptions of humanities practices miss distinctions in departmental norms. Given the impact that departmental norms play in promotion and tenure decisions, some departments may be predisposed to expanded team research efforts. On the other hand, a targeted approach to culture change may be required to nurture interdisciplinary research in other departments.

The theme of culture change is also essential when considering the impact of rank and career phase on the inclusion of faculty members in collaborative research. The data in the pilot set indicate that faculty members publish more collaboratively later in their careers. If organizations want to foster the inclusion of early-career faculty members in institutional team research efforts, then changes in standards for promotion and tenure or other incentives may be 
required. Further work is needed to determine the most significant barriers for early-career collaboration.

If prior collaboration is an indicator of collaborative readiness and success, as other studies indicate, then there are departments and individual faculty members that should be approached as initial partners in team research endeavors. These individuals can also be key collaboration brokers for colleagues and facilitate the development of shared conceptual frameworks through their experience. With librarians' expertise in cross-discipline dialogue and ability to connect people and resources, we can play a similar role. Through our knowledge of scholarly creation practices and tools such as expertise systems, we can identify opportunities for expansion of team research, including a deeper integration of humanities scholars in "grand challenges" research.

\section{Acknowledgments}

It is strange to write an article on coauthorship without a coauthor, but this study was not done without support from colleagues. This research was conducted during a professional development leave sponsored by the University of Minnesota Libraries. I would also like to thank my library colleagues for their help with conceptualizing the project, understanding the Experts@Minnesota system, and guidance on using APIs and manipulating XML: Steven Braun, Kate McCready, Joshua Bishoff, Amy Neeser, and Connie Hendrick.

Jennie M. Burroughs is Director for Arts and Humanities at the University of Minnesota Libraries in Minneapolis; she may be reached by e-mail at: jburroug@umn.edu.

\section{Notes}


1. Barry Bozeman and Craig Boardman, Research Collaboration and Team Science, SpringerBriefs in Entrepreneurship and Innovation (Cham, Switz.: Springer, 2014), 1.

2. C. P. Snow, The Two Cultures, ACLS [American Council of Learned Societies] Humanities E-Book XML ed. (Cambridge: Cambridge University Press, 2008).

3. Stefan Wuchty, Benjamin F. Jones, and Brian Uzzi, "The Increasing Dominance of Teams in Production of Knowledge," Science 316, 5,827 (2007): 1036.

4. Monica E. Bulger, Eric T. Meyer, Grace De la Flor, Melissa Terras, Sally Wyatt, Marina Jirotka, Katherine Eccles, and Christine McCarthy Madsen, "Reinventing Research? Information Practices in the Humanities" (London: Research Information Network, April 2011) (accessed June 11, 2015), http://www.rin.ac.uk/our-work/using-and-accessing-informationresources/information-use-case-studies-humanities, 7.

5. Lynne Siemens, Richard Cunningham, Wendy Duff, and Claire Warwick, "A Tale of Two Cities: Implications of the Similarities and Differences in Collaborative Approaches within the Digital Libraries and Digital Humanities Communities," Literary and Linguistic Computing 26, 3 (2011): 336.

6. Bulger, Meyer, De la Flor, Terras, Wyatt, Jirotka, Eccles, and Madsen, "Reinventing Research?" 52.

7. Wuchty, Jones, and Uzzi, “The Increasing Dominance of Teams," 1037.

8. Office of the Senior Vice President for Academic Affairs and Provost, University of Minnesota, "A Strategic Plan for the University of Minnesota Twin Cities" (Minneapolis: University of Minnesota, October 2014) (accessed February 26, 2015), https://drive.google.com/file/d/0Bw9rfbrwA2NWVUxjR3U3emtzR0U/view?usp=sharing, 27, 30. 
9. Ibid., 32.

10. Jenny M. Lewis, Sandy Ross, and Thomas Holden, “The How and Why of Academic Collaboration: Disciplinary Differences and Policy Implications,” Higher Education 64, 5 (2012): 702 .

11. Diana Rhoten and Andrew Parker, "Risks and Rewards of an Interdisciplinary Research Path,” Science 306, 5704 (2004): 2046.

12. University of Minnesota Libraries, A Multi-Dimensional Framework for Academic Support: A Final Report (Minneapolis: University of Minnesota Libraries, June 2006) (accessed July 16, 2015), http://purl.umn.edu/5540, 30, 75.

13. Lisa Mooney Smith, Knowledge Transfer in Higher Education: Collaboration in the Arts and Humanities (Houndmills, U.K.: Palgrave Macmillan, 2012), 109.

14. Daniel Stokols, Shalini Misra, Richard P. Moser, Kara L. Hall, and Brandie K. Taylor, “The Ecology of Team Science: Understanding Contextual Influences on Transdisciplinary Collaboration," American Journal of Preventive Medicine 35, 2 (2008): S106.

15. Jonathon N. Cummings and Sara Kiesler, "Who Collaborates Successfully? Prior Experience Reduces Collaboration Barriers in Distributed Interdisciplinary Research," Proceedings of the 2008 ACM [Association for Computing Machinery] Conference on Computer Supported Cooperative Work (2008), 443.

16. Willard McCarty, Humanities Computing (New York: Palgrave Macmillan, 2005), 11. 17. Ibid., 12.

18. John Unsworth, “The Humanist: 'Dances with Wolves' or 'Bowls Alone'?” presented at "Scholarly Tribes and Tribulations: How Tradition and Technology Are Driving Disciplinary Change," Association of Research Libraries conference, Washington, DC (October 17, 2003), 
accessed June 11, 2015, http://www.arl.org/about/tour-this-website/1207-the-humanist-danceswith-wolves-or-bowls-alone\#.VXmgLs-6e70, 6.

19. Ibid.

20. J. Sylvan Katz and Ben R. Martin, “What Is Research Collaboration?” Research Policy 26, 1 (1997): 2.

21. Ibid., 3 .

22. Wuchty, Jones, and Uzzi, “The Increasing Dominance of Teams,” 1037.

23. Jenny M. Lewis, Connecting and Cooperating: Social Capital and Public Policy (Sydney, Australia: UNSW [University of New South Wales] Press, 2010), 172.

24. Lisa Spiro, "Collaborative Authorship in the Humanities," Digital Scholarship in the Humanities: Exploring the Digital Humanities, blog (August 21, 2009), accessed July 7, 2015, https://digitalscholarship.wordpress.com/2009/04/21/collaborative-authorship-in-thehumanities/.

25. Julianne Nyhan and Oliver Duke-Williams, "Joint and Multi-Authored Publication Patterns in the Digital Humanities," Literary and Linguistic Computing 29, 3 (2014): 388.

26. Ibid., 393.

27. Jeremy Birnholtz, Shion Guha, Y. Connie Yuan, Geri Gay, and Caren Heller, "CrossCampus Collaboration: A Scientometric and Network Case Study of Publication Activity across Two Campuses of a Single Institution," Journal of the American Society for Information Science and Technology 64, 1 (2013): 167.

28. University of Minnesota Libraries, A Multi-Dimensional Framework for Academic Support, 24,30 .

29. Ibid., 22. 
30. Spiro, "Collaborative Authorship in the Humanities"; Nyhan and Duke-Williams, "Joint and Multi-Authored Publication Patterns in the Digital Humanities." 